\title{
Les espaces publics parisiens à l'épreuve de la démocratie locale
}

\author{
Antoine Fleury, Paris
}

\section{Introduction}

Il faut attendre les années 1980 pour que les rues et les places apparaissent comme un type d'espace présentant un certain nombre de caractéristiques propres, à la fois «espace de médiation, vecteur de vie sociale» et espace de «valeurs, symboles et signes de la culture urbaine» (Plan Urbain 1988: 111). Pendant les décennies précédentes, gérées comme un objet fonctionnel et technique, elles avaient été réduites à un simple espace de circulation. Ce changement progressif s'est fait à la faveur de nouvelles représentations de la ville, d'une part dans le champ des sciences sociales, plus sensibles aux rites de la vie ordinaire et aux formes de la socialité, d'autre part au sein des milieux professionnels où se redéploie un urbanisme culturaliste (BETIN 2001). Les «luttes urbaines» et plus généralement l'émergence d'une «nouvelle culture urbaine» participent également de ce mouvement à l'issue duquel le terme d'espace public s'impose pour désigner cet objet à part entière que sont les rues et les places (Tomas 2001).

Au cours des années 1980 et 1990, les pouvoirs publics font progressivement de cette catégorie l'un des éléments clés de leur action sur la ville. Né dans les villes danoises, hollandaises et suisses, ce renouvellement de l'action publique s'est ensuite diffusé dans toute l'Europe (Dessouroux 2006; Olagnier 2003). Ainsi a-t-on voulu «traiter les symptômes d'une crise sociale urbaine» par des espaces publics censés «développer les sentiments d'appartenance des individus à une même 〈cités» (Lussault 2003: 333). Un lien fort est alors établi avec la crise de la démocratie (HABERMAS 1962). L'action publique se fixe non seulement comme objectif de rétablir le lien distendu entre les citadins, mais aussi entre les citadins et le politique, faisant des espaces publics un «instrument de conversion politique» (Toussaint \& Zimmermann 2001: 88). L'affirmation récente de la démocratie locale est venue renforcer ce mouvement, en associant les habitants et les acteurs locaux à la production des espaces publics.

En s'appuyant sur le cas des espaces publics à Paris intra-muros, cet article se propose de caractériser les modes de production contemporains de l'espace public mais aussi de poser un regard critique sur cette évolution. Il s'agira dans un premier temps de présenter les résultats d'investigations menées entre 2003 et 2007 (Fleury 2007), en explicitant les modes de produc- tion actuels, leurs différents acteurs et leur généalogie. Dans un second temps, ces résultats seront interrogés au regard de la spécificité des espaces publics dans un contexte métropolitain. Il s'agira de prendre part au débat qui traverse actuellement les sciences sociales, entre les tenants du déclin et ceux de la renaissance des espaces publics (WIEGANDT 2006), en soulignant les contradictions et les risques sous-tendus par un renouvellement des modes de production qui privilégie l'échelon local.

\section{Posture et méthode}

Au-delà de la diversité de leurs formes, les espaces publics s'individualisent au sein de l'espace urbain par leur ouverture au public. Ils sont façonnés par des acteurs multiples, que ce soit par leurs pratiques spatiales ou par leurs décisions et leurs stratégies, ainsi que par l'interaction qui s'établit entre ces différents acteurs. Le choix d'une telle définition a conduit à combiner, sur le terrain, l'observation des formes et des pratiques de l'espace public, l'étude des jeux d'acteurs, et enfin, l'analyse de données de contexte.

Cet article s'appuie en particulier sur une série de 70 entretiens menés d'une part avec des élus ou leurs conseillers, d'autre part avec des techniciens de la Direction de la voirie et des déplacements (DVD), de la Direction des espaces verts et de l'environnement (DEVE) et de la Direction de l'urbanisme (DU) de la Ville de Paris, au niveau parisien et au niveau des arrondissements. Un certain nombre d'entretiens ont également été conduits avec des militants associatifs et des citoyens impliqués dans la démocratie locale, complétés par l'observation de plusieurs conseils de quartier. Enfin, un corpus de documents diversifié a été constitué (p.ex. documents officiels, compte rendus de réunions publiques, résultats de concertation, articles de presse, sites Internet) et analysé principalement de manière qualitative.

\section{Produire les espaces publics avec la société civile}

Les espaces publics parisiens ont longtemps été conçus selon une approche technique, à l'échelle de la ville. Tout en modifiant leur politique, les pouvoirs publics se sont progressivement ouverts à d'autres acteurs (Figure 1). Aujourd'hui, le mode d'association varie de la simple information à la participation, en passant par la concertation, pour reprendre une échelle issue de la sociologie des organisations (ARNSTEIN 1969). 


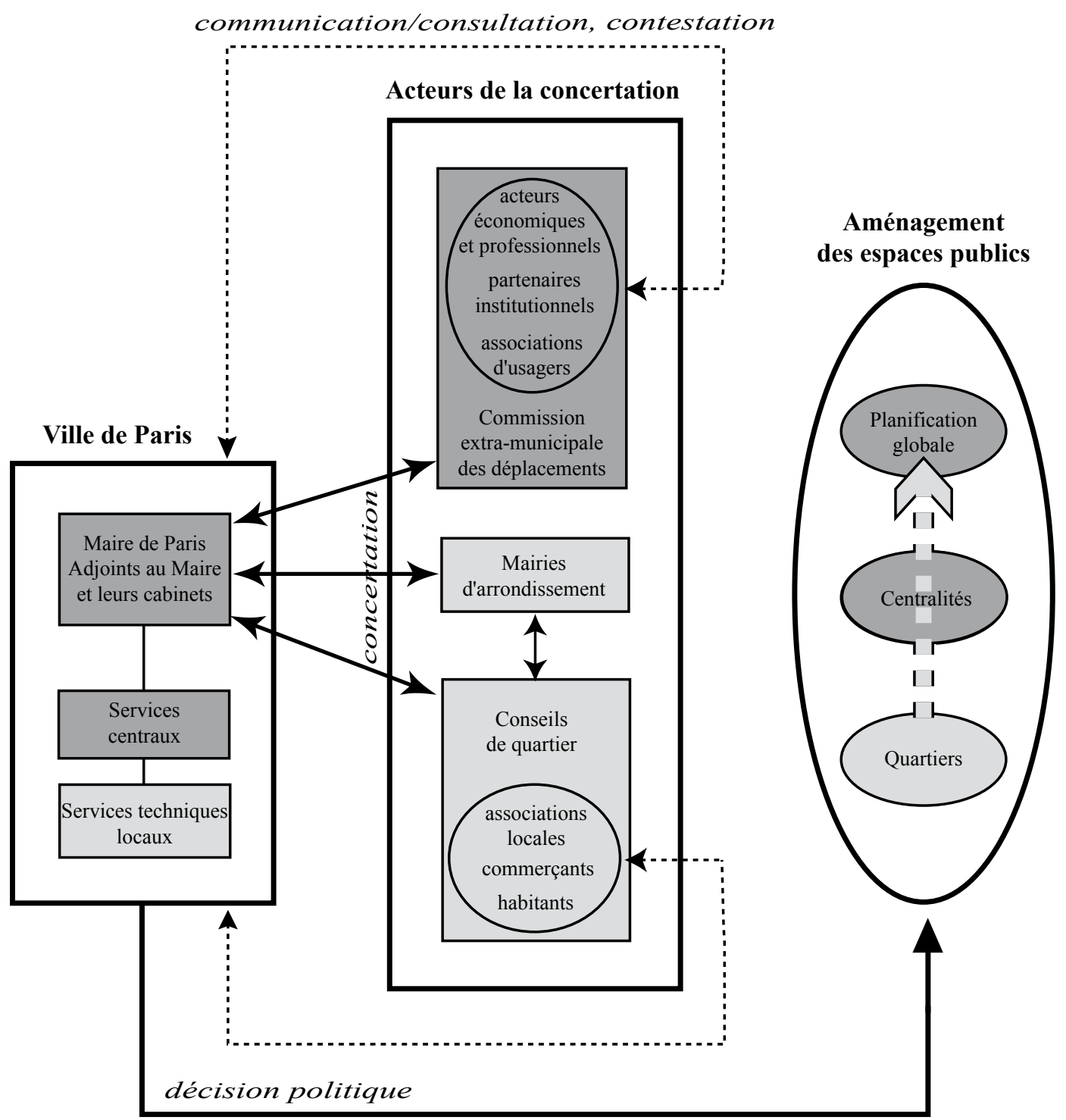

Fig. 1: Les acteurs de l'aménagement des espaces publics à Paris dans les années 2000 Actors of public space development in Paris during the 2000's Akteure der Gestaltung öffentlicher Räume in Paris seit 2000 Conception: A. FLEURY

\subsection{Une ouverture progressive de l'administration parisienne}

La politique des transports menée pendant les Trente Glorieuses, très favorable à l'automobile, est contestée dès les années 1960. De moins en moins motorisée et en voie d'embourgeoisement, la population accentue sa pression sur les élus par le biais d'associations dont le nombre va croissant (FLonNEAU 2005). Les grands projets d'infrastructures conduisent même à des «luttes urbaines» dans les années 1970. Mais pendant la décennie suivante, malgré l'abandon de ces projets et la requalification d'espaces publics emblématiques, 
l'amélioration de la circulation reste au cœur de la politique municipale. De plus, les Parisiens ne disposent toujours pas de tribunes pour se faire entendre en dehors des élections. Certes, deux types d'organes consultatifs sont créés suite à la Loi du 2 mars 1982 . A l'échelle de Paris, les représentants de la Mairie, de la Préfecture de police, de la Chambre de commerce et d'industrie de Paris (CCIP) ainsi que les associations d'usagers participent à la Commission extramunicipale des transports. Les Comités d'initiative et de consultation d'arrondissement (CICA) réunissent quant à eux les représentants des associations locales. Néanmoins, si ces instances sont régulièrement amenées à débattre des espaces publics, le statut à part de Paris - avec une Préfecture de police aux compétences larges, une administration centrale puissante et des arrondissements aux marges de manœuvre réduites - restreint leur rôle et elles demeurent de surcroît peu consultées.

Bien que reconduite, la majorité municipale de droite sort affaiblie des élections de 1995. Elle doit désormais rendre des comptes de manière plus régulière. En même temps qu'elle redéfinit la politique des déplacements de manière à favoriser davantage les modes de déplacement alternatifs à l'automobile, la municipalité revalorise donc les organes consultatifs hérités des années 1980, et notamment la Commission extramunicipale des transports, rebaptisée Commission extra-municipale des déplacements (CEMD). Ainsi le Plan Vélo a-t-il été élaboré en étroite collaboration avec les associations, de même que certains grands projets d'aménagement comme celui des Grands Boulevards. Si cette concertation s'est limitée aux associations, l'ouverture des pouvoirs publics à de nouveaux acteurs et la prise en compte de leurs points de vue apparaissent incontestablement comme une nouveauté par rapport à la période précédente.

Depuis 2001, avec l'arrivée à la Mairie d'une majorité de gauche, ces outils de concertation demeurent d'actualité, mais la démocratie locale est approfondie, intégrant plus en amont la concertation voire la participation. Les espaces publics tiennent d'autant plus une place de choix dans ce dispositif que de nombreux projets d'aménagement sont lancés avec la volonté de diminuer fortement la circulation automobile et de mieux partager l'espace public au profit des «circulations douces» (piétons, cyclistes, rollers) et des transports en commun. Les paragraphes suivants donnent à voir la diversité des outils utilisés pour produire les espaces publics dans le cadre de cette nouvelle politique.

\subsection{L'information et la consultation de la population}

Depuis 2001, la nouvelle municipalité a renforcé sa communication en direction de la population. Des campagnes d'affichage et de distribution conséquentes ont été organisées au moment de la mise en place des projets d'aménagement d'espaces publics, ainsi que de nombreuses réunions publiques et des expositions. Le site Internet de la Mairie possède une section dédiée aux déplacements, qui énumère les acteurs impliqués et propose toute une série d'informations sur les «grands projets». Des sites spécifiques ont en outre été créés pour l'élaboration du Plan local d'urbanisme (PLU) puis du Plan des déplacements de Paris (PDP), avec tous les éléments d'information par exemple sur le processus de concertation, les comptes rendus des réunions publiques, des forums de discussion.

Au moment de ces concertations, la Mairie a aussi commandé des enquêtes à des instituts de sondage, qui ont été l'occasion de consulter directement le public sur la planification des aménagements d'espaces publics. Plusieurs questions ont été posées à ce sujet, par exemple sur la végétation dans la ville, les déplacements et le stationnement, l'animation des rues. Les Parisiens interrogés se sont massivement prononcés pour l'aménagement de nouveaux espaces verts et la «végétalisation» des rues; ils ont également majoritairement considéré comme une priorité les aménagements favorisant les transports en commun et les piétons; enfin, la quasi-totalité se dit favorable à la protection du commerce de proximité (MAIRIE De PARIs 2005).

\subsection{La concertation avec les acteurs associatifs et économiques}

En ce qui concerne l'élaboration des projets dont l'impact dépasse les quartiers où ils s'inscrivent, la CEMD, à laquelle participent les mairies d'arrondissement, les opérateurs de transports, la Région, la Préfecture de police, les associations d'usagers, le Syndicat des taxis et la Chambre de commerce, constitue la clé de voûte de la concertation. C'est le cas en premier lieu des lignes de bus en site propre dans le cadre du programme Mobilien. Depuis 2001, les trois étapes d'un projet font ainsi l'objet d'une concertation dans ce cadre: diagnostic de la ligne, propositions d'aménagement, programme retenu. Au sein de la CEMD s'est en second lieu préparée l'élaboration du Plan des déplacements de Paris (PDP). La commission a ainsi été saisie à chaque phase d'élaboration du projet, les différents acteurs étant alors informés et pouvant réagir aux mesures envisagées. Elle a également travaillé en commissions autour de plusieurs thématiques dont une sur les espaces publics. En 2006, phase finale de la concertation, chaque acteur a transmis un avis écrit synthétisant sa position.

Au niveau local, les Comités d'initiative et de consultation d'arrondissement (CICA) continuent à jouer un rôle important. Créés dans les années 1980 mais alors peu intégrés dans le processus de décision, ils ont été 
revalorisés pendant le mandat de JeAn Tibéri. C'est principalement dans ce cadre qu'est alors mise en œuvre la concertation concernant les «quartiers tranquilles» (i.e. «zones 30» avec aménagements paysagers). Aujourd'hui, le rôle des CICA a été approfondi. On y discute des problèmes de déplacements et de cadre de vie rencontrés à l'échelle des quartiers. Ainsi, les projets Mobilien ne sont pas seulement débattus dans le cadre de la CEMD, mais aussi dans le cadre des CICA, avec une présentation du diagnostic et des projets. Les associations de quartier et les représentants des commerçants peuvent ainsi prendre connaissance des projets et les discuter, voire proposer des modifications.

\subsection{La concertation avec les habitants et les acteurs locaux}

Après 2001, les conseils de quartier sont généralisés. La Loi Démocratie et Proximité de 2002 leur donne un cadre légal et ils deviennent rapidement des instances où les acteurs locaux peuvent s'exprimer sur les problèmes qu'ils rencontrent dans les espaces publics ou critiquer les projets de la Mairie. Leur création constitue un changement d'autant plus important qu'en parallèle, les arrondissements ont vu s'accroître leurs compétences, notamment en matière de concertation.

\subsubsection{De la concertation à la participation}

Depuis 2002, l'essentiel de la concertation précédant l'aménagement des espaces publics de proximité se fait dans le cadre des conseils de quartier. C'est principalement le cas pour les projets de «quartiers verts» (qui ont pris le relais des «quartiers tranquilles»). Les Sections territoriales de voirie (STV), services déconcentrés de la Direction de la voirie, réalisent un diagnostic sur le périmètre adopté par les mairies d'arrondissement. Le diagnostic est ensuite présenté dans le cadre d'une réunion publique au cours de laquelle le conseil de quartier est invité à faire des suggestions. La mairie d'arrondissement et la STV construisent alors des propositions qui sont présentées une nouvelle fois au conseil de quartier. Au cours de chaque réunion, la présentation générale de l'élu précède l'intervention du technicien, pour finir sur les questions et remarques du public; un compte rendu est élaboré qui circule entre les services. Malgré tout, les procédures de concertation restent plus consultatives que participatives: les conseils de quartier sont surtout sollicités pour signaler les points sensibles en termes de sécurité routière ou de localisation du mobilier urbain.

Cependant, ces conseils s'affirment aussi comme une force de proposition. Certains ont réussi à obtenir le réaménagement d'espaces publics, participant largement à la définition des projets. De plus, sur le modèle des budgets participatifs développés à Porto Allegre, un budget est dédié aux conseils de quartier depuis 2003. Ces derniers peuvent acquérir des biens consommables, notamment pour les repas de quartier. Ils disposent d'un «fonds de participation» pour financer des micro-projets: p.ex. création d'emplacements réservés pour les deux-roues, pose de barrières pour sécuriser les cheminements piétons, réfection des trottoirs, amélioration de l'éclairage public. Par ailleurs, les budgets alloués peuvent être mutualisés entre conseils de quartier pour des projets plus ambitieux.

\subsubsection{De l'échelon local à l'échelon parisien}

Les conseils de quartier interviennent aussi de plus en plus dans la planification et les programmes d'échelle urbaine, à côté de la CEMD. Ainsi, les méthodes de concertation utilisées dans le cadre du programme Mobilien ont évolué depuis 2004. Jusqu'à cette date, la concertation a été menée dans le cadre de la CEMD, la réunion publique n'intervenant qu'une fois le consensus acquis entre les acteurs institutionnels, associatifs et économiques. Or cette méthode a conduit à une situation de blocage. C'est pourquoi, depuis 2004, la Mairie de Paris diffuse le diagnostic puis les hypothèses aux conseils de quartiers, ajoutant un nouveau lieu dans le processus de concertation (Figure 1). Les conseils de quartier travaillent sur les documents puis une seconde réunion est organisée, au cours de laquelle élus et experts écoutent les réactions du public.

La Mairie de Paris a aussi appelé les conseils de quartier à faire des propositions concrètes pour élaborer le Plan local d'urbanisme (PLU) puis le Plan des déplacements de Paris (PDP), successivement en 2003 et en 2005. Les espaces publics ont été érigés comme un sujet majeur de préoccupation lors de la concertation sur le PLU. Ainsi, pour l'ensemble des conseils de quartier qui se sont exprimés, $47 \%$ des propositions ont concerné les rues et les places (Figure 2). L'expression forte de cette préoccupation en faveur des espaces publics est venue légitimer les mesures déjà prises par la Mairie - justifiant à ses yeux un renforcement des dispositifs dans ce domaine - même si, au moment de la concertation pour le PDP notamment, les habitants et associations ont aussi pointé certaines incohérences et insuffisances.

\section{Les espaces publics entre débat public et appropriation locale}

Les effets de ce nouveau mode de production sont éminemment contradictoires. L'aménagement des espaces publics, autrefois dominé par la logique administrative et technique, est désormais mis en débat. Mais ce nouveau mode de production, qui subit les limites intrinsèques de la démocratie locale, remet en cause certaines spécificités de l'espace public métropolitain. 


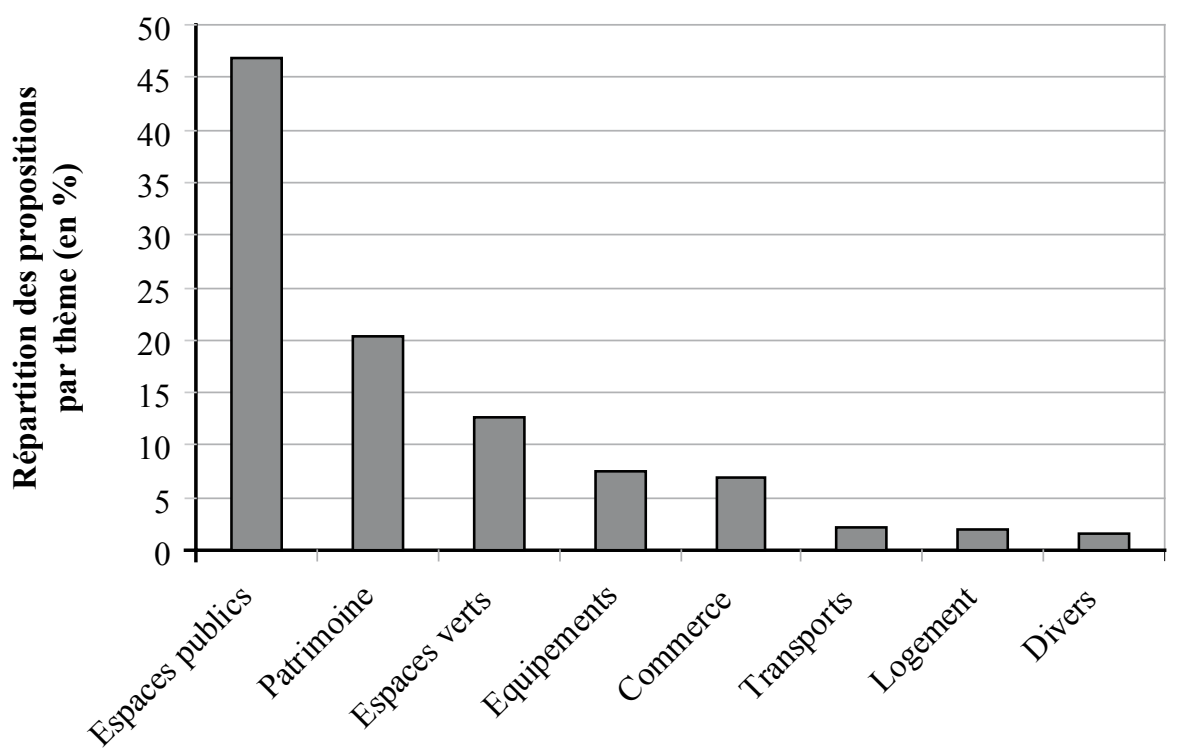

Thèmes des propositions

Fig. 2: Les propositions des conseils de quartier parisiens lors de la concertation sur le Plan local d'urbanisme (PLU) en 2003

Suggestions of Parisian local councils during the consultation process concerning the City Plan in 2003

Vorschläge der Pariser Quartiersräte im Abstimmungsprozess bezüglich des Stadtplans im Jahr 2003

Source: Mairie de Paris/Direction de l'urbanisme, Mission PLU

\subsection{Espaces publics et débat public}

Par le biais de la question de la circulation, l'aménagement des espaces publics a certes été l'objet de vifs débats publics tout au long du $\mathrm{XX}^{\mathrm{e}}$ siècle (FlonneAu 2005), opposant société civile et pouvoirs publics. Mais les débats décisifs se tenaient dans les «arènes formelles de décision» (FrançoIs \& NEveu 1999) où ils avaient un caractère «feutré et opaque» pendant la période de forte homogénéité politique (RULLIER 2004: 20). De plus, ils étaient très influencés par les experts. En rupture avec cette tradition, la part du politique dans l'aménagement des espaces publics a été revalorisée dans les années 2000. Non seulement les élus définissent désormais la politique mise en œuvre par leurs services à l'échelon parisien, mais les maires d'arrondissement ont gagné de nouvelles compétences, en particulier celle d'organiser la concertation.

Si c'est en réaffirmant leur capacité à faire des choix que les élus rendent possible le débat, le choix de la concertation vient véritablement institutionnaliser ce dernier. La concertation telle qu'elle est organisée en amont des projets suit en effet peu ou prou les trois principes de la démocratie délibérative (BLONDIAUX \&
Sintomer 2002). Elle crée en premier lieu les conditions de possibilité du principe de publicité. Les réunions sont en effet annoncées par courrier, par affichage, par la presse ou sur Internet. La concertation crée en second lieu les conditions de possibilité du principe d'inclusion. Les lieux de la concertation sont en effet ouverts au plus grand nombre: acteurs associatifs, habitants et acteurs économiques. Enfin, le choix de la concertation s'accompagne d'un développement accru des modalités d'information et de communication, créant les conditions de possibilité du principe d'argumentation. Ainsi, les pouvoirs publics se sont beaucoup investis pour former les citoyens aux enjeux d'aménagement, par exemple en clarifiant les objectifs ou en explicitant les contraintes techniques.

$\mathrm{Au}$ regard des comptes rendus de réunion et des observations menées dans plusieurs conseils de quartier, le débat public apparaît globalement comme une réalité (FLEURY 2007). Les participants commentent et critiquent les projets, apportent des idées et échangent avec les élus et les experts. Néanmoins, si le débat est une réalité au sein de certains conseils de quartier, il peut être moins riche voire inexistant dans d'autres 
conseils de quartier. Il dépend en effet de la volonté et/ou de la capacité des élus d'arrondissement d'une part, des membres du conseil de quartier d'autre part, de créer les conditions du débat. Enfin, si une procédure commune se dessine, des différences importantes apparaissent entre des arrondissements aux couleurs politiques différentes.

\subsection{Appropriation du débat par les résidents et les classes moyennes}

Le jeu de la démocratie locale fait que l'on consulte principalement les franges les plus favorisées de la population, qui sont celles qui s'investissent davantage dans la démocratie locale (Blondiaux 2004). C'est d'autant plus le cas que la surreprésentation de ces catégories de population se renforce à Paris (CLERVAL 2008) et que de surcroît les aménagements sont sensiblement plus nombreux dans les quartiers en voie de gentrification (FLEURY 2007). A l'inverse, les catégories les plus défavorisées sont rarement présentes dans les réunions publiques. Certes, des dispositifs particuliers permettent à ces habitants de s'impliquer dans la vie publique locale, comme c'est le cas dans les Grands projets de renouvellement urbain (GPRU). Mais ils ne concernent que les quartiers identifiés comme défavorisés. Enfin, d'autres usagers de l'espace public ne sont que rarement consultés: usagers de passage, simples visiteurs réguliers ou salariés travaillant dans le secteur. Il en va de même pour les usagers que l'on considère souvent comme «indésirables» (Belina 2003), dont les préoccupations ne pourraient être prises en compte que par le biais de dispositifs adaptés qui demeurent tout aussi rares.

Des entretiens avec les acteurs publics et du suivi des débats au sein de plusieurs conseils de quartier, il ressort que les demandes formulées par les résidents renvoient principalement à une exigence de protection - notamment face à la circulation - doublée d'une exigence d'embellissement ou de préservation du cadre de vie. De ce fait, il n'est pas étonnant que les espaces publics constituent l'un des thèmes les plus discutés dans les instances de la démocratie locale, aux dépens $\mathrm{du}$ logement ou des transports par exemple (Figure 2). Pour les résidents impliqués dans la démocratie locale, c'est leur qualité de vie qui compte le plus, et les espaces publics doivent être mis au service de cette dernière: on doit pouvoir se promener et faire ses achats en toute tranquillité, dans des lieux esthétiquement irréprochables. Si ces exigences peuvent renvoyer à un intérêt collectif à l'échelle du quartier, d'autres renvoient à des intérêts particuliers. Parce qu'ils remettent en cause le cadre de vie et les habitudes des riverains, l'aménagement de couloirs de bus ou l'abattage d'arbres sont ainsi activement contestés, de même que la réduction du nombre de places de stationnement, ce qui ne va pas sans contradictions puisque les mêmes riverains militent aussi souvent pour des trottoirs plus larges.

\subsection{Pressions des intérêts locaux et fermeture des espaces publics}

Faut-il aller à l'encontre de l'avis des habitants, au risque de les décevoir, ou à l'inverse, partager le pouvoir, au risque d'une perte de cohérence? Si les pouvoirs publics parisiens cherchent globalement à concilier intérêts locaux et intérêt général dans leurs décisions, certains élus n'arbitrent pas voire tranchent en faveur d'intérêts locaux, ce que bon nombre de techniciens de la Ville de Paris n'hésitent pas à dénoncer. Ces élus semblent ainsi agir dans une logique électorale, le choix de produire les espaces publics à l'échelon local venant renforcer une tendance propre à la gestion de la ville dans une démocratie. Parmi les conséquences sur la nature même de l'espace public, deux semblent particulièrement préoccupantes parce qu'elles tendent vers une fermeture de ce dernier.

\subsubsection{L'échelon local contre la ville?}

Les intérêts locaux peuvent entrer en contradiction avec ce que fait la ville dans son ensemble. C'est tout particulièrement le cas en ce qui concerne le patrimoine. Dans les réunions de concertation, les idées sur la qualité du paysage et sur le patrimoine de voirie sont difficiles à faire passer, parce qu'elles vont souvent à l'encontre des intérêts locaux. La prise en compte de ces intérêts contribue même à une complexification des aménagements, et notamment à la multiplication des objets dans l'espace public, rendant celui-ci de plus en plus hétérogène. Le cas des lignes de Mobilien en témoigne: une série d'aménagements différenciés ont été mis en place en fonction des quartiers traversés, rompant la continuité paysagère de l'axe emprunté. La multiplicité des périmètres et la complexité de la concertation aboutissent de surcroît à des problèmes de suture évidents, soulevant la question du manque d'harmonisation entre les aménagements.

La pression des habitants et des associations de quartier pose également un autre problème fondamental qui est celui de la fluidité, indispensable au bon fonctionnement de la ville. Un technicien nous a même déclaré que «les habitants veulent un quartier vert où personne ne peut venir sauf eux, des sens interdits qu'ils peuvent prendre...». Les intérêts locaux entrent alors clairement en conflit avec l'intérêt général: peuton concevoir une ville où les quartiers ne pourraient être traversés que par leurs habitants? La question se pose d'autant plus pour les voies du réseau principal dont le réaménagement se fait désormais aussi en concertation avec les riverains. Or, le grand axe est certes un élément de leur cadre de vie, mais c'est aussi un lieu de passage, de sociabilité et de commerce à l'échelle de la métropole. S'il est important de repen- 
ser les relations entre les différentes échelles de l'espace public, on ne saurait donc faire de ce dernier un espace public exclusivement de proximité.

\subsubsection{De l'espace public au «paysage public»?}

A Paris comme dans de nombreuses métropoles européennes, «la mise en valeur des villes à travers une politique d'espaces publics» apparaît en adéquation «avec les besoins et les désirs [de citadins] jeunes et bien dotés en capital culturel» (BASSAND et al. 2001: 108). Ainsi, sous la pression des résidents, les espaces publics de proximité deviennent une composante essentielle des «paysages urbains que peuvent «consommer〉 les classes moyennes et moyennes supérieures» (Sмiтh 2003: 58). Ils se muent en une scène que les classes dominantes se réapproprient par leurs pratiques (p.ex. de sociabilité, d'achat) en même temps qu'elles marquent symboliquement, à travers les aménagements paysagers qu'elles encouragent, leur possession du sol et le contrôle qu'elles exercent sur les relations sociales (MitcheLL 1996).

Si cette tendance peut être interprétée comme une renaissance, en termes de formes comme de pratiques, elle incarne aussi un nouvel ordre urbain où les espaces publics apparaissent de moins en moins comme des lieux de diversité ou de conflits sociaux. Ils se muent en espaces de grande qualité esthétique peu propices aux usages traditionnels; ils peuvent même constituer une barrière symbolique pour des groupes qui par ailleurs s'investissent peu dans la concertation. Les aménagements viennent alors renforcer un processus d'exclusion déjà constaté au niveau résidentiel (CleRval 2008). Et l'exclusion de se faire plus radicale encore quand il s'agit de groupes considérés comme «indésirables» (Belina 2003). C'est d'ailleurs en analysant la place des sans-logis dans les politiques urbaines que Mitchell (1997) a proposé la notion de «paysage public». Si la tendance est moins engagée à Paris que dans certaines métropoles nord-américaines, les aménagements dissuasifs tendent malgré tout à s'y diffuser sous la pression de résidents réclamant par exemple la suppression d'un banc occupé par un sans-logis ou la pose de barrières pour éloigner des groupes de jeunes.

\section{Conclusion}

Ces dernières années, s'il y a renaissance des espaces publics en contexte métropolitain, ce n'est pas qu'en termes de formes d'aménagement, même si c'est sans nul doute la dimension la plus visible. Les nouveaux modes de production ont également participé de cette renaissance. En effet, les logiques administrative et technique ont laissé place à des logiques plus démocratiques et les compétences du citadin sont désormais largement mobilisées.
Néanmoins, cette évolution tend à remettre en cause l'idéal d'ouverture à tous. D'une part, l'affirmation de la démocratie locale a renforcé la tendance à produire les espaces publics dans la proximité, contribuant à un brouillage des échelles de la ville. Car les espaces publics ne peuvent se réduire à l'échelon local, de surcroît au sein des espaces centraux d'une grande métropole, fréquentés par des milliers de personnes venues de toute l'agglomération voire de plus loin. D'autre part, si ce choix de la proximité se justifie en partie par le développement de la mobilité et du polycentrisme, qui bouleverse les repères des citadins, il s'apparente aussi à une privatisation rampante des espaces publics au profit des résidents qui, par leurs pressions sur les élus, cherchent à transformer ces derniers à leur image. Ainsi le processus de privatisation des espaces publics ne se réduit-il pas uniquement à l'intervention croissante du secteur privé (Glasze 2001). En définitive, à Paris comme dans d'autres contextes métropolitains en Europe (BAssand et al. 2001; Dessouroux 2006; WiEgANDT 2006), les deux points de vue - apparemment antagonistes - de la renaissance et du déclin s'entrecroisent de manière complexe et diversifiée.

Il ressort enfin de cette étude que la production des espaces publics ne peut faire l'impasse d'une articulation forte des acteurs et des échelles de la métropole. Pour contrebalancer l'affirmation de la démocratie locale, le rôle de l'administration doit sans doute être réaffirmé. En s'appuyant sur ses compétences techniques, celle-ci doit veiller au respect de l'intérêt général. Si les services ne peuvent se substituer aux élus pour définir l'intérêt général, leur mission est désormais de veiller à ce que les intérêts particuliers ne l'emportent pas sur l'intérêt collectif, les intérêts locaux sur l'intérêt général. En même temps doivent être approfondis les dispositifs de concertation en direction d'un plus large public, en intégrant les habitants les plus pauvres, les «indésirables» et tout simplement les usagers de passage.

\section{Bibliographie}

Arnstein, S.R. (1969): A ladder of citizen participation. - In: Journal of the American Planning Association 35, 4: 216-224.

Bassand, M., Compagnon, A., Güller, P., Joye, D. \& V. STEIN (2001): Vivre et créer l'espace public. - Lausanne: Presses Polytechniques et Universitaires Romandes.

Belina, B. (2003): Evicting the undesirables. The idealism of public space and the materialism of the bourgeois State. - In: Belgeo 1: 47-62.

Betin, C. (2001): La construction de l'espace public: le cas de Lyon. - In: Géocarrefour 76, 1: 47-54.

Blondiaux, L. (2004): L'idée de démocratie participative: enjeux, impensés et questions récurrentes. - Montréal: Institut d'études internationales, Confé- 
rence prononcée à l'Université du Québec à Montréal, http://www.ieim.uqam.ca/IMG/pdf/Blondiaux-conf1novembre-2004.pdf 31.10.2009.

Blondiaux, L. \& Y. Sintomer (2002): L'impératif délibératif. - In: Politix 57: 17-35.

Clerval, A. (2008): La gentrification à Paris intramuros: dynamiques spatiales, rapports sociaux et politiques publiques. - Thèse de doctorat en géographie, Université de Paris 1.

Dessouroux, C. (2006): La production des espaces publics dans la ville contemporaine. Enjeux, acteurs et logiques de gestion et d'aménagement dans trois communes de la région Bruxelles-Capitale. - Thèse de doctorat en géographie, Université Libre de Bruxelles.

Fleury, A. (2007): Les espaces publics dans les politiques métropolitaines. Réflexions au croisement de trois expériences: de Paris aux quartiers centraux de Berlin et Istanbul. - Thèse de doctorat en géographie, Université de Paris 1.

Flonneau, M. (2005): Paris et l'automobile, un siècle de passions. - Paris: Hachette.

FrançoIs, B. \& E. Neveu (1999): Introduction: Pour une sociologie politique des espaces publics contemporains. - In: FrançoIs, B. \& E. Neveu (éds): Espaces publics mosaïques: acteurs, arènes et rhétoriques des débats publics contemporains. - Rennes: Presses Universitaires de Rennes: 13-58.

Glasze, G. (2001): Privatisierung öffentlicher Räume? Einkaufszentren, Business Improvement Districts und geschlossene Wohnkomplexe. - In: Berichte zur Deutschen Landeskunde 75, 2-3: 160-177.

Habermas, J. (1962): L'espace public. Archéologie de la publicité comme dimension constitutive de la société bourgeoise. - Paris: Payot.

Lussault, M. (2003): Espace public. - In: LÉvy, J. \& M. Lussault (éds): Dictionnaire de la Géographie et de l'espace des sociétés. - Paris: Belin: 333-336.

Mairie de PARIs, Direction GÉNÉrale de L'INFormation ET DE LA COMMUNICATION/IPSOS (éd.) (2005): Consultation sur le PLU. Construire avec vous l'avenir de Paris. Vos réponses, http://www.paris.fr/fr/Urbanisme 23.08.2006 (rubrique Plan Local d'Urbanisme).

Mitchell, D. (1996): Political violence, order, and the legal construction of public space: Power and the public forum doctrine. - In: Urban Geography 17, 2: 152-178.

MrtcheLL, D. (1997): The annihilation of space by law: the roots and implications of anti-homeless laws. - In: Antipode 29: 303-335.

Olagnier, P.-J. (2003): Voirie et espace public: mise en regard de pratiques d'aménagement à Barcelone, Londres et Paris. - Thèse de doctorat en géographie et aménagement, Université de Paris 1.

Plan Urbain (1988): Espaces publics. - Paris: La Documentation Française.

RULLIER, B. (2004): La démocratie à la parisienne. - In: Pouvoirs 110: 19-34.
Sмiтh, N. (2003): La gentrification généralisée: d'une anomalie locale à la «régénération» urbaine comme stratégie urbaine globale. - In: BIDOU-ZaCHARIASEN, C. (éd.): Retours en ville. - Paris: Descartes \& Cie: 45-72. Tomas, F. (2001): L'espace public, un concept moribond ou en expansion? - In: Géocarrefour 1:75-84.

Toussaint, J.-Y. \& M. ZimmermanN (2001): L'espace public et l'espace du public. Politique et aménagement. - In: Toussaint, J.-Y. \& M. Zimmermann (éds): User, observer, programmer et fabriquer l'espace public. - Lausanne: Presses Polytechniques et Universitaires Romandes: 73-91.

WiEgandT, C.-C. (éd.) (2006): Öffentliche Räume - öffentliche Träume. Zur Kontroverse über die Stadt und ihre Gesellschaft. - Berlin, Münster: Liт Verlag.

\section{Résumé: Les espaces publics parisiens à l'épreuve de la démocratie locale}

En deux décennies, les espaces publics sont devenus l'un des éléments clés de l'action publique sur la ville. A mesure que de nouvelles formes d'aménagement ont émergé, de nouveaux modes de production ont aussi vu le jour. Les pouvoirs publics se sont ouverts à d'autres acteurs, que ce soient les associations d'usagers, les associations d'habitants ou les habitants eux-mêmes. Cette évolution a été relativement lente à Paris, dans un contexte politico-institutionnel très particulier. Aujourd'hui, il existe plusieurs modes d'association, de la simple information à la participation, en passant par la consultation de la population ou la concertation au moment des projets d'aménagement. Il convient cependant de poser un regard critique sur ce renouvellement. Certes l'aménagement des espaces publics, autrefois dominé par la logique administrative et technique, peut désormais être publiquement débattu. Mais ce nouveau mode de production demeure soumis aux limites intrinsèques de la démocratie locale. Les intérêts locaux pèsent fortement sur les choix d'aménagement. Or, ces intérêts sont principalement des intérêts de résidents, en particulier ceux qui sont issus des classes moyennes et supérieures. Ce faisant, les espaces publics se muent en «paysages publics», en contradiction avec ce qui fait la ville dans son ensemble.

Mots-clés: espaces publics, aménagement urbain, démocratie locale, métropole, Paris

\section{Abstract: Public space and local democracy in Paris}

Over the last 20 years, public urban policies in Paris have increasingly acted upon public spaces. In the process, development patterns and modes of public action have been changed. New actors have emerged in this field, from individual residents to users' and residents' associations. Given the specific politicalinstitutional context of the French capital, this evolu- 
tion has been relatively slow. New modes of decisionmaking have been implemented, ranging from mere information or consultation to more elaborate participation mechanisms. These new modes of public space discourse have yet to be evaluated critically, as they raise a number of issues. Although the development of public space has become a public matter, it is still limited by the very constraints of local democracy. Local interests, for example, increasingly shape processes of change, reflecting mainly the interests of middle and upper class residents for whom «public landscapes» are being created that often contradict other dimensions of the city.

Keywords: public spaces, urban planning, local democracy, metropolis, Paris

\section{Zusammenfassung: Öffentliche Räume und Lokaldemokratie in Paris}

In den letzten zwanzig Jahren wurden öffentliche Räume zentrale Aspekte öffentlicher Stadtpolitik, und neue Stadtgestaltungsformen entstanden. Neue Akteure traten ins Kräftefeld städtebaulicher Planung, z.B. Nutzer- und Einwohnervereine sowie die Einwohner selbst. Diese Entwicklung fand in Paris relativ zögernd statt, wohl auf Grund der für die französische Hauptstadt charakteristischen politisch-institutionellen Konstellation. Heutzutage gibt es vielerlei Formen der Absprache zwischen Behörden und Bürgern: Sie reichen von der einfachen Information und Konsul- tation bis hin zur Abstimmung und Bürgerbeteiligung. Doch diese neuen Interaktionsformen bringen auch neue Probleme mit sich, die kritisch betrachtet werden müssen. Wenngleich die Gestaltung öffentlicher Räume nunmehr in der Öffentlichkeit diskutiert wird, unterliegt sie gleichzeitig den Einschränkungen der lokalen Demokratie. So haben Lokalinteressen - hauptsächlich diejenigen der besserverdienenden Einwohner - einen immer gewichtigeren Einfluss auf Gestaltungsentscheidungen. Für diese Einwohner entstehen somit regelrechte «public landscapes», die mit anderen Dimensionen der Stadt in Widerspruch stehen.

Schlüsselwörter: Öffentliche Räume, Stadtentwicklung, Lokaldemokratie, Metropole, Paris

Dr. Antoine Fleury, Centre National de la Recherche Scientifique (CNRS), UMR Géographie-cités, 13, rue du Four, F-75006 Paris, France.

e-mail : afleury@parisgeo.cnrs.fr

\section{Manuskripteingang/received/manuscrit entré le} 5.7.2009

Annahme zum Druck/accepted for publication/accepté pour l'impression: 26.12 .2009 